\title{
Reversible Addition Fragmentation Chain Transfer (RAFT) Polymerization of 4-Vinylbenzaldehyde
}

\author{
Guorong Sun, Chong Cheng, and Karen L. Wooley ${ }^{\star}$ \\ Center for Materials Innovation and Department of Chemistry, Washington University in Saint Louis, \\ One Brookings Drive, Saint Louis, MO 63130-4899
}

\begin{abstract}
The direct reversible addition fragmentation chain transfer (RAFT) polymerization of 4vinylbenzaldehyde (VBA) was established as a new synthetic method for the preparation of welldefined poly(vinylbenzaldehyde) (PVBA), a polymer having reactive aldehyde side chain substiuents. RAFT polymerization of VBA was investigated using $S$-1-dodecyl- $S^{\prime}-\left(\alpha, \alpha^{\prime}\right.$-dimethyl$\alpha$ "-acetic acid)trithiocarbonate (DDMAT) as chain transfer agent (CTA) and 2,2'-azobis (isobutyronitrile) (AIBN) as initiator in 1,4-dioxane or 2-butanone at $70-75^{\circ} \mathrm{C}$ for $7.5-22.5 \mathrm{~h}$. With 45-76\% of monomer conversion, the resulting PVBA had well controlled number-average molecular weight $\left(M_{n}\right)$ and low polydispersity $(P D I<1.17)$. The living characteristic of the RAFT polymerization process was confirmed by the linearity between the $M_{n}$ values of PVBA and monomer conversions. Well-defined PVBA was further used as a macromolecular chain transfer agent (macroCTA) in RAFT polymerization of styrene (St), and a block copolymer PVBA- $b$-PSt with relatively low polydispersity $(P D I=1.20)$ was successfully synthesized.
\end{abstract}

Construction of polymers with highly reactive functionalities that allow for further diverse functional group transformation (FGT) is an emerging research area in modern polymer chemistry. ${ }^{1}$ Among these polymers, polymers bearing aldehyde functionalities are attractive because aldehydes are among the most reactive substrates for FGT under mild reaction conditions. ${ }^{2}$ Conventional radical polymerization of aldehyde-functionalized monomers has been studied since $1950 \mathrm{~s},{ }^{3}$ however, the resulting polymers lacked well-defined structures, due to the non-living nature of the polymerization technique. ${ }^{4-7}$ Anionic polymerization provided an alternative synthetic pathway for the preparation of aldehyde-functionalized polymers, ${ }^{8-13}$ but the stringent polymerization conditions and tedious preparation procedures (protection of monomer, polymerization, and deprotection of the resulting polymers were required) significantly restrict the applicability of this synthetic route.

To efficiently prepare well-defined aldehyde-functionalized polymers, we investigated living radical polymerization of aldehyde-functionalized monomers. In the past decade, reversible addition fragmentation chain transfer (RAFT) polymerization has been developed and demonstrated as a powerful tool in living radical polymerization. ${ }^{14,15}$ Relative to other living radical polymerization techniques, one important advantage of RAFT is that it provides facile and homogenous living polymerization systems applicable for a wide variety of monomers under relatively mild reaction conditions. In this communication, we report our recent work of RAFT polymerization of 4-vinylbenzaldahyde (VBA), as a new methodology for the construction of well-defined aldehyde-functionalized polymers (Scheme 1).

\footnotetext{
*Corresponding author: phone, (314) 935-7136; fax, (314) 935-9844; e-mail: klwooley@ artsci.wustl.edu
} 
Several synthetic pathways for the synthesis of VBA have been reported in the literature, including transition metal-catalyzed cross-coupling of 4-bromobenzaldehyde with ethylene or vinyl reagents, Grignard addition to $N, N$-dimethyl formamide (DMF) followed by acid hydrolysis, and Wittig olefination. ${ }^{5-7}$ We prepared VBA by Wittig olefination of 4(diethoxymethyl)benzaldehyde, a commercially available mono-protected benzene-1,4dicarboxaldehyde, followed by deprotection (Scheme 1, a slight modification from Dhal's method $^{6}$ ), because this reliable synthetic pathway had satisfactory yield ( $82 \%$ over 2 -steps) with feasible scale-up and simple work-up protocols. As demonstrated by Dhal et al., ${ }^{6}$ radical polymerization of the intermediate monomer (diethyl acetal-protected styrene) followed by deprotection can also yield PVBA. However, we prefer to directly introduce aldehyde functionalities by using VBA as a (co)monomer because of our intention to avoid deprotection of polymers and also the key concern that the post-polymerization deprotection step may result in deconstruction of useful functionalities presented on the polymers, including the terminal RAFT functionalities.

RAFT polymerization of VBA was then conducted, and as a result, well-defined PVBA was synthesized (Table 1). Relative to other chain transfer agents (CTAs) for RAFT polymerization systems, $S$-1-dodecyl- $S^{\prime}$-( $\alpha, \alpha^{\prime}$-dimethyl- $\alpha$ "-acetic acid)trithiocarbonate (DDMAT) ${ }^{16}$ can be prepared readily and has less unfavorable odor and therefore, it was used as the CTA in our study $\left([\mathrm{VBA}]_{0} /[\mathrm{CTA}]_{0}=100 / 1.0\right)$. A typical thermal initiator 2,2'-azobis(isobutyronitrile) $(\mathrm{AIBN})$ was used as initiator $\left([\mathrm{CTA}]_{0} /[\mathrm{AIBN}]_{0}=1.0 / 0.1-0.2\right)$, and the polymerization temperature was chosen at $70-75^{\circ} \mathrm{C}$ to maintain a suitable decomposition rate of AIBN to provide initiating radicals. Because of the poor solubility of PVBA in its monomer, either 1,4dioxane or 2-butanone was used as the polymerization solvent, and 2-butanone was further found as a better solvent than 1,4-dioxane for PVBA. ${ }^{17}$ For each trial, the monomer conversion was determined by ${ }^{1} \mathrm{H}-\mathrm{NMR}$ analysis of the final polymerization solution based on comparison of the integration area of resonances of aldehyde protons of PVBA at $9.8 \mathrm{ppm}$ and the integration area of resonances of aldehyde protons of VBA at $10.0 \mathrm{ppm}$ corresponding to the aldehyde proton signals from both polymer and monomer, respectively. PVBA was obtained by precipitation of the final polymerization solution twice into cold pentane and drying under vacuum. 18

The well-defined structure of PVBA was verified by ${ }^{1} \mathrm{H}$ NMR and Gel Permeation Chromatography (GPC) analyses (Figure 1). As shown in Figure 1(a), the integration area ratio of aldehyde protons $v s$. aromatic protons in PVBA was 1.00:2.12:2.03, illustrating the essential absence of side reactions on the aldehyde functionality of VBA during the RAFT process and the quantitative presence of aldehyde functionalities in PVBA. The presence of the $\omega$ trithiocarbonate RAFT functionality in PVBA was indicated by its ${ }^{1} \mathrm{H}$ NMR resonances at 0.88 and $3.22 \mathrm{ppm}$ (for $\mathrm{CH}_{3}$ and $\mathrm{SCH}_{2}$, respectively), and the ${ }^{1} \mathrm{H}$ NMR resonances of the benzylic proton of the terminal VBA unit at $4.84 \mathrm{ppm}$ with an integration area ratio of 1.00:1.93 with the $\mathrm{SCH}_{2}$ at $3.22 \mathrm{ppm}$. The number-average degree of polymerization was obtained by ${ }^{1} \mathrm{H}$ NMR spectroscopy $\left(D P_{N M R}\right)$, by comparing the intensity of the benzylic proton of the terminal VBA unit at $4.84 \mathrm{ppm}$ with that of the aldehydic proton signal at $9.8 \mathrm{ppm}$, and was found to agree with the number-average degree of polymerization obtained by GPC $\left(D P_{G P C}\right)$ within the error range (for example for sample entry 5 (Table 1), the $D P_{N M R}$ and $D P_{G P C}$ values were 78 and 75, respectively). Molecular weight and molecular weight distribution data for the PVBA samples were determined by GPC using THF as eluent (Figure 1(b)). All PVBA samples have not only excellent agreements between the GPC and calculated (based on monomer conversion) molecular weights but also monomodal molecular weight distributions with low polydispersties (PDI = 1.06-1.17), indicating well-controlled chain growth and very high chain transfer efficiency of the trithiocarbonate RAFT functionality in the polymerization process. 
To confirm the living characteristic of this RAFT polymerization system, the relationships between polymer molecular weights and monomer conversions were studied and polymerization kinetics were also investigated (Figure 2). During polymerization, aliquots were withdrawn from the reaction vessel at predetermined times and analyzed by ${ }^{1} \mathrm{H}-\mathrm{NMR}$ spectroscopy for determination of the monomer conversions and by GPC for determination of the polymer molecular weights and polydispersities. As shown in Figure 2(a), excellent linear agreement $\left(R^{2}=0.9976\right)$ between polymer molecular weight and monomer conversion was obtained, and the resulting polymers also maintained narrow molecular weight distributions $(P D I=1.07$ to 1.11). Such results verified the living characteristics of the RAFT polymerization process. As shown in Table 1, similar to conventional radical polymerization of $\mathrm{VBA},{ }^{4,5,7 \mathrm{a}}$ RAFT polymerization of VBA proceeded relatively slowly. Figure 2(b) depicts the kinetic plot of the polymerization. Pseudo first-order kinetics were maintained at the initial polymerization stage $(\sim 4 \mathrm{~h})$, and then the kinetics deviated from linearity. Because AIBN has a short half-life of $c a .3 \mathrm{~h}$ at $75^{\circ} \mathrm{C}$, such retardation in polymerization might essentially be the result of the decreased radical concentration due to the depletion of AIBN.

The living characteristics of RAFT polymerization has allowed ready preparation of a broad variety of block copolymers. ${ }^{19} \mathrm{We}$ also investigated the chain extension of the well-defined PVBA having terminal RAFT functionality by RAFT polymerization as a synthetic method for the preparation of aldehyde-functionalized block copolymers. Although copolymerizations of vinylbenzaldehydes with styrene have already been extensively studied, ${ }^{5,7}$ so far there is no literature report on the synthesis of block copolymers bearing pendant aldehyde functionalities by radical polymerization. Using PVBA $\left(M_{\mathrm{n}, \mathrm{GPC}}=10,300 \mathrm{Da}, P D I=1.17\right)$ as macro-CTA and AIBN as initiator, RAFT polymerization of styrene ([St $]_{0} /[\text { macro-CTA }]_{0} /$ $\left.[\mathrm{AIBN}]_{0}=150 / 1.0 / 0.1\right)$ was conducted at $60^{\circ} \mathrm{C}$ in 2-butanone $(70 \mathrm{vol} \%)$. The polymerization was allowed to proceed for $12 \mathrm{~h}$, and $17 \%$ conversion of styrene was obtained, as measured by ${ }^{1} \mathrm{H}$ NMR spectroscopy. As shown in Figure 1(c), the formation of diblock copolymer PVBA- $b$-PSt $\left(M_{\mathrm{n}, \mathrm{GPC}}=12,500 \mathrm{Da}, P D I=1.19\right)$ by chain extension from the PVBA-based macro-CTA was verified by GPC analysis. Moreover, the good agreement between the experimental and calculated molecular weights $\left(M_{\mathrm{n}, \text { calcd }}=12,900 \mathrm{Da}\right)$ and the mono-modal molecular weight distribution of the PVBA- $b$-PSt illustrates the quantitative chain transfer efficiency of the PVBA-based macro-CTA.

In summary, we have established RAFT homopolymerization of 4-vinylbenzaldehyde as a new and facile synthetic method for the preparation of PVBAs with predictable molecular weights and low polydispersities. The chain extension from PVBA for the preparation of block copolymers also has been explored and PVBA- $b$-PSt has been constructed. To our knowledge, this is the first example of block copolymer bearing multiple aldehydes by direct polymerization without protected monomer, which was possible by applying radical polymerization. We believe that these homo- and block (co)polymers can serve as templates for feasible access of polymers with more complex architectures or conjugates with bio-active substrates for biological and medicinal applications via well-developed carbonyl chemistry.

\section{Supplementary Material}

Refer to Web version on PubMed Central for supplementary material.

\section{Acknowledgment}

Financial support from the National Heart Lung and Blood Institute of the National Institutes of Health as a Program of Excellence in Nanotechnology (U01 HL080729) is gratefully acknowledged. We also acknowledge postdoctoral fellowship support from Unilever Corporation (CC). 


\section{References and Notes}

(1). Takemoto, K.; Ottenbrite, RM.; Kamachi, M., editors. Functional Monomers and Polymers. Vol. 2nd ed.. Marcel Dekker; New York: 1997.

(2). Larock, RC. Comprehensive Organic Transformations - A Guide to Functional Group Transformations. Vol. 2nd ed.. Wiley-VCH; New York: 1999.

(3). Wiley RH, Hobson PH. J. Poly. Sci 1950;5:483-486.

(4). Mulvaney JE, Chang DM. Macromolecules 1980;13:240-243.

(5). Pichot C, Charleux B. Makromol. Chem 1992;193:187-203.

(6)(a). Dhal PK. Macromol. Rep 1993;A30:93-99. (b) Dhal PK, Khisti RS. Chem. Mater 1993;5:16181623.

(7)(a). Heinenberg M, Menges B, Mittler S, Ritter H. Macromolecules 2002;35:3448-3455. (b) Heinenberg M, Ritter H. Macromol. Chem. Phys 2002;203:1804-1810.

(8). Hirao A, Ishino Y, Nakahama S. Makromol. Chem 1986;187:141-147.

(9). Hirao A, Nakahama S. Macromolecules 1987;20:2968-2972.

(10). Ishizone T, Kato R, Ishino Y, Hirao A, Nakahama S. Macromolecules 1991;24:1449-1454.

(11). Ishizone T, Sugiyama K, Hirao A, Nakahama S. Macromolecules 1993;26:3009-3018.

(12). Ishizone T, Sueyasu N, Sugiyama K, Hirao A, Nakahama S. Macromolecules 1993;26:6976-6984.

(13). Ishizone T, Utaka T, Ishino Y, Hirao A, Nakahama S. Macromolecules 1997;30:6458-6466.

(14). Rizzardo, E.; Chiefari, J.; Mayadunne, RTA.; Moad, G.; Thang, SH. Controlled/Living Radical Polymerization. Progress in ATRP, NMP, and RAFT. Matyjaszewski, K., editor. Vol. 768. American Chemical Society; Washing, DC: 2000. p. 278-296.

(15). For comprehensive reviews of RAFT polymerization, see(a)Moad G, Rizzardo E, Thang SH. Aust. J. Chem 2005;58:379-410.410(b)Perrier S, Takolpuckdee P. J. Polym. Sci. Part A: Polym. Chem 2005;43:5347-5393.5393(c)Moad G, Rizzardo E, Thang SH. Aust. J. Chem 2005;59:669-692.692

(16)(a). Lai JT, Filla D, Shea R. Macromolecules 2002;35:6754-6756.

(17). As far the co-solvents have been reported including DMF, 1,4-dioxane, benzene, and 2-butanone. However, we found the PVBA, especially those with relatively high molecular weight (DP > 60), do not dissolve well in either DMF or benzene.

(18). Initially we used cold methanol for precipitation. After drying, ${ }^{1} \mathrm{H}-\mathrm{NMR}$ spectra of the polymer clearly showed the partial formation of dimethyl acetal (extra signals at $\delta=3.3$ and $5.3 \mathrm{ppm}$ ) because the terminal carboxylic acid functionality catalyzed the "re-protection" reaction of the aldehyde.

(19)(a). Lei P, Wang Q, Hong J, Li Y. J. Polym. Sci., Part A: Polym. Chem 2006;44:6600-6606. (b) Bussels R, Bergman-Gottgens C, Klumperman B, Meuldijk J, Koning C. J. Polym. Sci., Part A: Polym. Chem 2006;44:6419-6434. (c) Nguyen TLU, Eagles K, Davis TP, Barner-Kowollik C, Stenzel MH. J. Polym. Sci., Part A: Polym. Chem 2006;44:4372-4383. (d) Lai JT, Shea R. J. Polym. Sci., Part A: Polym. Chem 2006;44:4298-4316. (e) Bilalis P, Pitsikalis M, Hadjichristidis N. J. Polym. Sci., Part A: Polym. Chem 2006;44:659-665. (f) Monteiro MJ. J. Polym. Sci., Part A: Polym. Chem 2005;43:5643-5651. (g) O’Reilly RK, Joralemon MJ, Hawker CJ, Wooley KL. J. Polym. Sci., Part A: Polym. Chem 2006;44:5203-5217. (h) Germack DS, Harrisson S, Brown GO, Wooley KL. J. Polym. Sci., Part A: Polym. Chem 2006;44:5218-5228. 


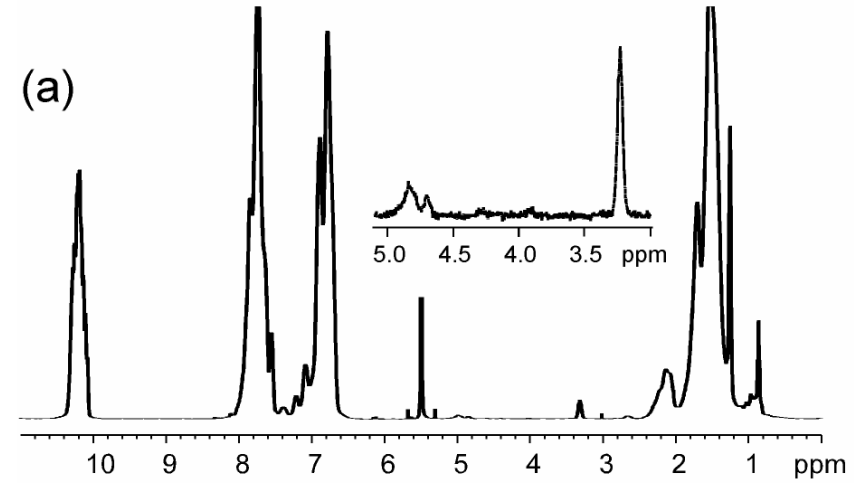

(b)
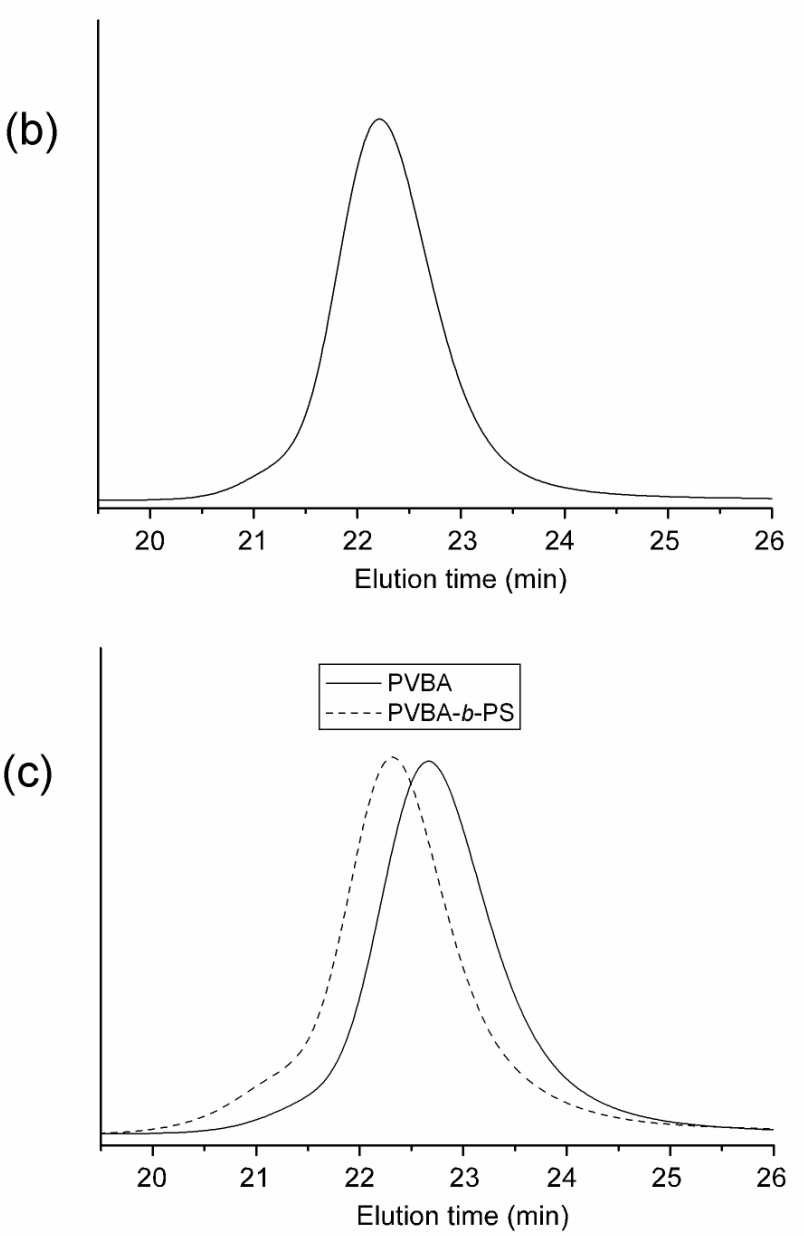

Figure 1.

Reversible addition fragmentation chain transfer polymerization of 4-vinylbenaldehyde: (a) ${ }^{1} \mathrm{H}-\mathrm{NMR}$ spectrum of poly(vinylbenzaldehyde) (PVBA) (500 MHz, (Methylene chloride)$d_{2}$; Table 1 entry 5). (b) Gel Permeation Chromatography (GPC) curve for PVBA (Table 1 entry 5). (c) GPC curve for PVBA (Table 1 entry 2) and Poly(vinylbenzaldehyde)- $b$-Poly (styrene) (polymerization conditions: $[\text { Styrene }]_{0} /[\text { macro-Chain Transfer Agent }]_{0} /[\text { Initiator }]_{0}=$ 150/1.0/0.1, 70 vol \% of 2-butanone, $60{ }^{\circ} \mathrm{C}, 12 \mathrm{~h}$ ). 

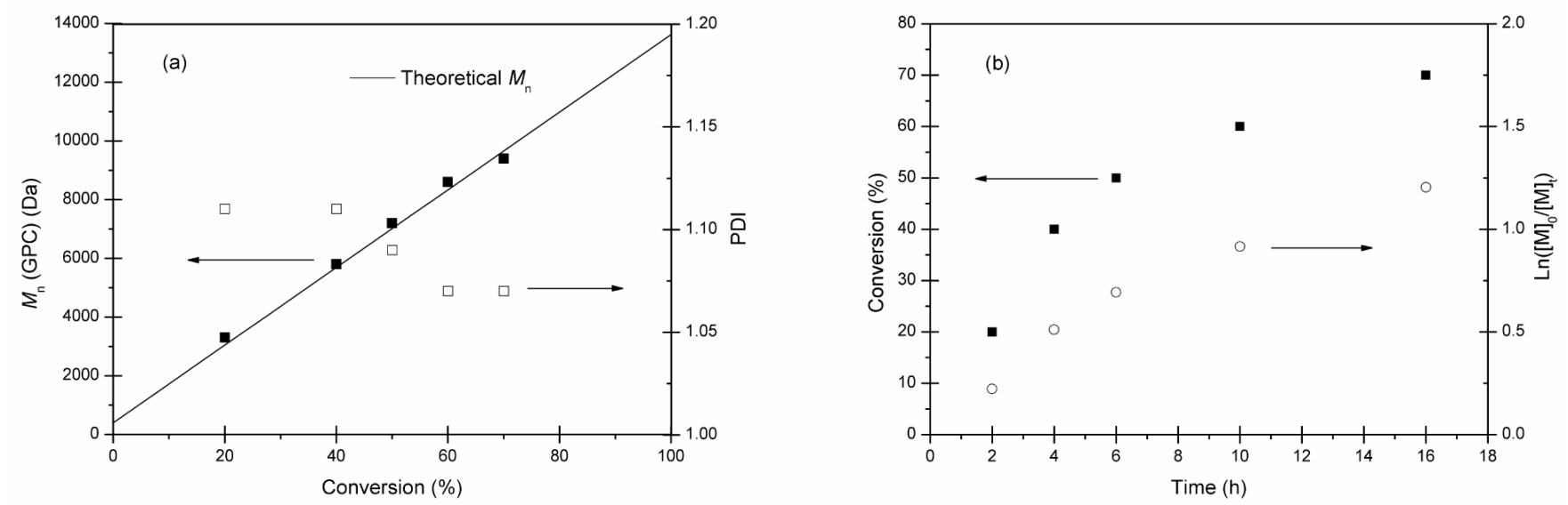

Figure 2.

Kinetic plots for reversible addition fragmentation chain transfer polymerization of 4vinylbenzaldehyde: (a) relationship of number-average molecular weight $\left(M_{n}, \boldsymbol{\square}\right)$ and polydispersity index $(P D I, \square)$ versus monomer conversion; (b) time dependence of monomer conversion $[\mathrm{M}]$ and $\ln \left([\mathrm{M}]_{0} /[\mathrm{M}]_{\mathrm{t}}\right)$. (polymerization conditions: $[\mathrm{VBA}]_{0} /[$ Chain Transfer Agent $]_{0} /[\text { [nitiator }]_{0}=100 / 1.0 / 0.1,50 \mathrm{vol} \%$ of 2 -butanone, $75^{\circ} \mathrm{C}$ ). 
<smiles>CCOC(OCC)c1ccc(C=O)cc1</smiles><smiles>C=Cc1ccc(C(OCC)OCC)cc1</smiles><smiles>C=Cc1ccc(C=O)cc1</smiles>

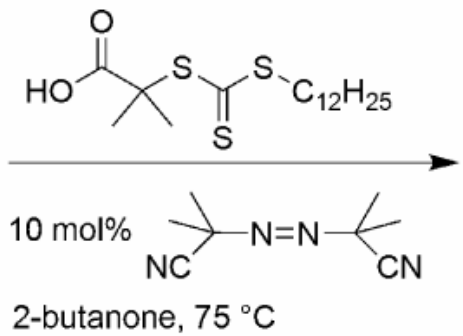<smiles>CCCCCCCCCCC(C)(CC(C)(C)C(=O)O)c1ccc(C=O)cc1</smiles><smiles>C=Cc1ccc(C(C)(C)N=NC(C)(C)C#N)cc1</smiles>

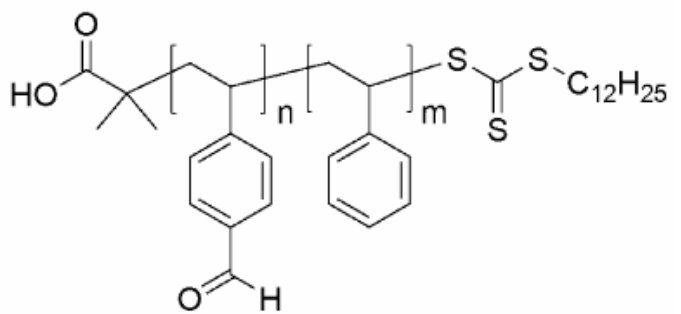

Scheme 1. 


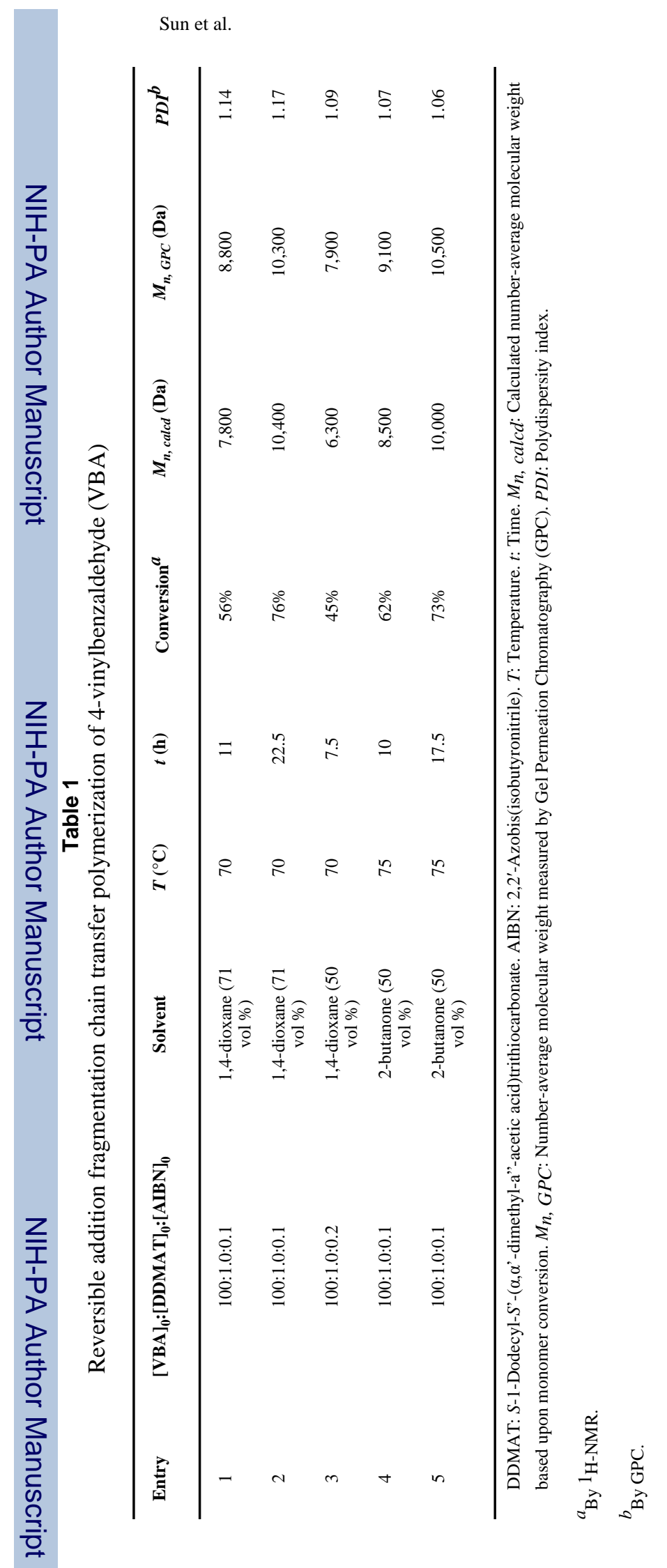

Macromolecules. Author manuscript; available in PMC 2008 December 8. 